\title{
Diseño de estribos abiertos en puentes de carretera obtenidos mediante optimización híbrida de escalada estocástica
}

\section{Design of open reinforced concrete abutments road bridges with hybrid stochastic hill climbing algorithms}

A. Luz ${ }^{(*)}, \underline{\text { V. Yepes }}{ }^{(*)}$, F. González-Vidosa ${ }^{(*)}$, J. V. Martí ${ }^{(*)}$

\section{RESUMEN}

Este artículo se ocupa del diseño automático de estribos abiertos de hormigón armado en puentes de carretera de coste mínimo, empleando para ello dos algoritmos híbridos de escalada estocástica con operadores de mutación basados en los algoritmos genéticos. Los algoritmos empleados se basan en el recocido simulado (SAMO) y en la aceptación por umbrales (TAMO). Ambos algoritmos se aplican a un estribo definido por 40 variables discretas. Se han comprobado ahorros económicos superiores al $18 \%$ respecto a un estribo de referencia de una altura de $9 \mathrm{~m}$ realmente construido, con diferencias pequeñas entre ambos algoritmos, del 0,5 \% a favor de SAMO. Además, se ha realizado un estudio paramétrico para alturas de estribo entre 6 y $15 \mathrm{~m}$ para diferentes tensiones admisibles del terreno que ofrece criterios de predimensionamiento a los proyectistas. Se ha comprobado, además, que el ahorro económico se localiza fundamentalmente en la zapata de estas estructuras.

Palabras clave: Hormigón estructural; optimización heurística; estribos; recocido simulado; aceptación por umbrales; diseño estructural; puentes.

\section{ABSTRACT}

This article deals with the minimum cost automatic design of reinforced concrete open abutments of road bridges, using a two-hybrid stochastic hill climbing algorithms with a neighborhood move based on the mutation operator from the genetic algorithms. These algorithms are based on the simulated annealing (SAMO) as well as on the threshold accepting procedure (TAMO). Both algorithms were applied to an open abutment which has 40 discrete variables. Savings have been found $18 \%$ compared to an abutment of $9 \mathrm{~m}$ in height really executed. SAMO improves by only $0.5 \%$ a similar run by TAMO. Additionally, the paper presents a parametric study of commonly used abutments from 6 to $15 \mathrm{~m}$ in height for different bearing conditions. Further, the results presented are of much value for the preliminary design of open abutments of road bridges. Finally, it is shown that cost savings are mainly located in the footing of these structures.

Keywords: Structural design; Heuristic optimization; Abutments; Simulated annealing; Threshold accepting; Structural design; Bridges.

(*) ICITECH - Universitat Politècnica de València, España.

Persona de contacto/Corresponding author: vyepesp@upv.es (V. Yepes)

Cómo citar este artículo/Citation: Luz, A., Yepes, V., González-Vidosa, F., Martí, J. V. (2015). Diseño de estribos abiertos en puentes de carretera obtenidos mediante optimización híbrida de escalada estocástica. Informes de la Construcción, 67(540): e114, doi: http:// dx.doi.org/10.3989/ic.14.089.

Licencia / License: Salvo indicación contraria, todos los contenidos de la edición electrónica de Informes de la Construcción se distribuyen bajo una licencia de uso y distribución Creative Commons Reconocimiento no Comercial 3.o. España (cc-by-nc). 


\section{INTRODUCCIÓN}

Los puentes de carretera constituyen infraestructuras esenciales en el desarrollo de las comunicaciones terrestres. Estas estructuras han sido objeto de optimización, especialmente en lo referente al diseño eficiente de tableros y pilas. Así, algunos trabajos recientes se han ocupado del diseño económico de tableros de vigas artesa (1) o pilas de puente (2). Sin embargo, a pesar de estos trabajos (3), se aprecia un vacío en el diseño completo de puentes reales (4), destacando la ausencia de estudios centrados en la optimización de los estribos. Los estribos sirven de apoyo al tablero y proporcionan soporte al terreno sobre el que descansa la calzada adyacente. Los tipos más comunes son los abiertos o los cerrados, en función de que las tierras lo atraviesen o queden contenidas por él, aunque también empiezan a ser habituales los estribos integrales (5).

La eficiencia en el diseño de las estructuras descansa fundamentalmente en la experiencia del proyectista. En efecto, el cálculo habitual comienza con un predimensionamiento y en una comprobación posterior de la estructura. Este diseño se puede mejorar en un proceso de prueba y error que, sin embargo, no garantiza la optimización de la estructura. Con todo, técnicas como RSD (Reinforcement Sizing Diagrams) permiten el armado óptimo de secciones de hormigón postesado (6). Estos procesos se agilizan actualmente con paquetes de servicios web para el análisis y diseño de estructuras (7). La resolución del problema se puede abordar mediante métodos de programación matemática (8), los cuales presentan limitaciones debido al crecimiento exponencial del tiempo de cálculo con el número de variables. Una revisión realizada en 1994 (9) indica cómo la mayoría de la optimización estructural se realizaba mediante programación matemática en problemas más bien teóricos, con una preponderancia del acero estructural frente al hormigón. Esta limitación explica el éxito de los algoritmos de optimización aproximada (10) como aquellos que emulan fenómenos naturales como los algoritmos genéticos (11), el recocido simulado (12) o la optimización por enjambres (13).

Las metaheurísticas irrumpieron con fuerza en la ingeniería estructural en los años setenta y ochenta, siendo preponde- rantes los algoritmos evolutivos (14). Estos algoritmos facilitan el diseño automatizado de las estructuras, puesto que partiendo de soluciones aleatorias, permiten guiar la búsqueda hacia soluciones factibles optimizadas. En las estructuras metálicas la función objetivo suele ser la minimización del volumen estructural (15) (16); lo cual no es aplicable al hormigón armado, donde las primeras aplicaciones son posteriores (17). En este sentido, nuestro grupo ha optimizado estructuras de hormigón tales como marcos (18) y bóvedas de paso inferior en carreteras (19), muros de contención (20) (21), tableros de vigas (22) o pilas de puente (23).

Siguiendo esta línea, el artículo se centra en el diseño automatizado de estribos abiertos, formados normalmente por una viga cabezal, dos pilares que lo soportan y una zapata corrida que reparte las cargas. La viga cabezal recibe el tablero y lo preserva del terraplén mediante un murete de guarda dispuesto sobre la viga y una aleta en vuelta en cada extremo. A estos elementos se añade una losa de transición entre el terraplén y el tablero, que se apoya en la viga cabezal. El estribo abierto presenta la ventaja que supone mejorar la visibilidad de los conductores de la vía inferior, además del ahorro de materiales que presenta respecto al estribo cerrado, sobre todo con terraplenes de cierta altura (24).

\section{DEFINICIÓN DEL PROBLEMA DE OPTIMIZACIÓN}

El problema consiste en minimizar el coste de un estribo abierto de hormigón armado representado por la expresión [1] incluyendo los costes indirectos (Tabla 1), satisfaciendo además las restricciones geométricas y de constructibilidad, así como los estados límite que se deben verificar, según la expresión [2].

$$
\begin{gathered}
C=f\left(x_{1}, x_{2}, \ldots, x_{n}\right)=\sum_{i=1}^{r} p_{i} \cdot m_{i}\left(x_{1}, x_{2}, \ldots, x_{n}\right) \\
g_{j}\left(x_{1}, x_{2}, \ldots . . x_{n}\right) \leq \mathrm{o} \\
x_{i} \in\left(d_{i 1}, d_{i 2}, \ldots, d_{i q_{i}}\right)
\end{gathered}
$$

Tabla 1. Unidades de obra y Precios unitarios.

\begin{tabular}{|c|l|r|}
\hline Ud. & \multicolumn{1}{|c|}{ Descripción de la unidad de obra } & $\begin{array}{r}\text { Precio unitario } \\
\text { (€) }\end{array}$ \\
\hline $\mathrm{m}^{3}$ & EXCAVACIÓN & 3,01 \\
\hline $\mathrm{m}^{3}$ & HORMIGÓN DE LIMPIEZA & 46,28 \\
\hline $\mathrm{m}^{2}$ & ENCOFRADO (zapata, cabezal y losa de transición) & 18,03 \\
\hline $\mathrm{m}^{2}$ & ENCOFRADO PILARES: DE ESPESOR CONSTANTE (trasdós pilares) & 18,63 \\
\hline $\mathrm{m}^{2}$ & ENCOFRADO PILARES: DE ESPESOR VARIABLE (laterales de pilares) & 24,64 \\
\hline $\mathrm{m}^{2}$ & ENCOFRADO DE MUROS (aletas, murete de guardia y tapas del cabezal) & 18,63 \\
\hline $\mathrm{kg}$ & ACERO B-5OO-S & 0,73 \\
\hline $\mathrm{m}^{3}$ & HORMIGÓN & 51,74 \\
\hline HA-25 & & 55,88 \\
\hline HA-30 & & 60,40 \\
\hline HA-35 & & 65,49 \\
\hline HA-40 & & 70,30 \\
\hline HA-45 & 75,11 \\
\hline HA-50 & & 4,81 \\
\hline $\mathrm{m}^{3}$ & RELLENO DE TIERRAS & \\
\hline
\end{tabular}


La estructura está definida por 40 variables que incluyen 9 geométricas, 29 de armado y 2 de materiales. Se han empleado los parámetros reflejados en la Tabla 2. El estribo de referencia se construyó en 1998 para un puente recto de vano continuo de la autovía A-58, tramo Santa Marta de Magasca - Cáceres. Se trata de un paso superior de dos vanos de $25 \mathrm{~m}$ de luz, un tablero de $8 \mathrm{~m}$ de ancho y un canto constante de $1 \mathrm{~m}$, apoyado sobre una pila central circular de $1 \mathrm{~m}$ de diámetro. Este estribo tiene una altura total de $9 \mathrm{~m}$ y una tensión admisible en el terreno de cimentación de o,5 MPa. En la Figura 1 se ha representado un esquema de dicho puente. El coste de este estribo, según los precios de la Tabla 1, es de 12.473,95 €. Se ha elegido esta estructura, además, por ser representativa dicha luz de un caso intermedio entre losas superiores macizas y aligeradas (25).

Tabla 2. Parámetros geométricos y de armados.

\begin{tabular}{|c|c|c|}
\hline Descripción & Nomenclatura & Valor \\
\hline \multicolumn{3}{|l|}{ Geométricos } \\
\hline Altura del estribo desde el terreno & Het & $7,50 \mathrm{~m}$ \\
\hline Longitud del cabezal & $L c$ & $8,00 \mathrm{~m}$ \\
\hline Ancho de la plataforma del tablero & plat & $7,00 \mathrm{~m}$ \\
\hline Distancia entre las dos reacciones & brazo & $6,20 \mathrm{~m}$ \\
\hline Distancia entre las reacciones y el murete de guarda & $b c 1$ & $0,70 \mathrm{~m}$ \\
\hline Altura del apoyo tablero & $h c 1$ & $1,30 \mathrm{~m}$ \\
\hline Ancho murete de guarda & $b c 2$ & $0,25 \mathrm{~m}$ \\
\hline Ancho del apoyo de la losa de transición & $b c 3$ & $0,30 \mathrm{~m}$ \\
\hline Espesor de firme sobre la losa de transición & hc2 & $1,00 \mathrm{~m}$ \\
\hline Canto de la losa de transición & hc3 & $0,30 \mathrm{~m}$ \\
\hline Altura entre la losa de transición y el cabezal (rótula) & he4 & $0,00 \mathrm{~m}$ \\
\hline Atura aletas sobre el cabezal & hc5 & $1,05 \mathrm{~m}$ \\
\hline Atura libre de neopreno y meseta inferior de apoyo & hc6 & $0,20 \mathrm{~m}$ \\
\hline Resguardo vertical de las tierras al cabezal & resg_v & $0,70 \mathrm{~m}$ \\
\hline Longitud de la aleta fuera del cabezal & la & $1,10 \mathrm{~m}$ \\
\hline Espesor de la aleta & ea & $0,25 \mathrm{~m}$ \\
\hline Longitud losa de transición sentido longitudinal puente & llt & $5,00 \mathrm{~m}$ \\
\hline Distancia lateral entre losa transición y aleta & $d l t$ & $0,25 \mathrm{~m}$ \\
\hline Espesor de firme bituminoso sobre la losa de transición & h_firme & $0,40 \mathrm{~m}$ \\
\hline Pendiente del relleno del terraplén & pte & 0,667 \\
\hline Profundidad del plano de cimentación & prof & $1,50 \mathrm{~m}$ \\
\hline Resguardo inferior entre la excavación y la zapata & resg_ex & $0,50 \mathrm{~m}$ \\
\hline Talud de la excavación en la zapata & talud_ex & 0,20 \\
\hline Espesor del hormigón de limpieza & ehlimp & $0,10 \mathrm{~m}$ \\
\hline \multicolumn{3}{|l|}{ De armados } \\
\hline Armadura bajo apoyos del tablero en cada dirección & Aapoyos_c & $9 Ø 16$ \\
\hline Armadura perimetral de zunchado en la zapata & $A p \_z$ & $\varnothing_{16 / 20 \mathrm{~cm}}$ \\
\hline
\end{tabular}

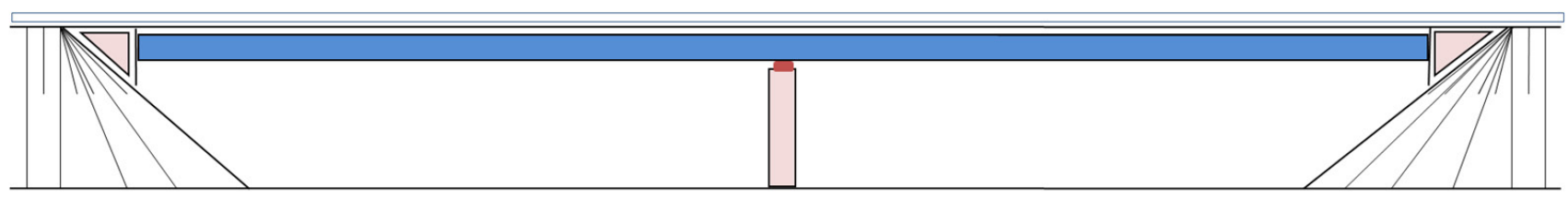

Figura 1. Esquema del alzado del puente de referencia de dos vanos continuos y $25 \mathrm{~m}$ de luz cada vano. 
La Figura 2 muestra las variables geométricas. La sección de la viga cabezal se define con las variables $h c$ y $b c o$; el resto son el canto inferior del pilar incrhip, el ancho de cada pilar $b p$ y la distancia entre pilares $d p$. La zapata se define en planta con la dirección de los dos pilares viz y $v t z$, transversalmente con incrlz y el canto con $h z$. La Figura 3 muestra las variables de armado, siendo 12 en el cabezal, 11 en los pilares y 6 en la zapata. Por último, hay que añadir dos variables correspondientes a los tipos de hormigón empleados en la zapata, $f c k \_z$, y en el alzado $f c k \_e$.

Se han contemplado las acciones permanentes debidas al peso propio del estribo y del tablero; las sobrecargas de trá- fico y viento; y las de valores constantes debidos al empuje de tierras sobre el estribo, a las deformaciones impuestas del tablero y a su pretensado. Se han considerado las acciones sobre la estructura de referencia: sobrecargas de $4 \mathrm{kN} / \mathrm{m}^{2}$ en el tablero, un carro de $600 \mathrm{kN} \mathrm{y} \mathrm{una} \mathrm{carga} \mathrm{de} 10 \mathrm{kN} / \mathrm{m}^{2}$ en el terraplén. Además, se tienen en cuenta un eje de dos ruedas del carro pisando sobre el murete de guarda (2 cargas de $100 \mathrm{kN}$ separadas $2 \mathrm{~m}$ ) y una carga lineal actuando sobre el apoyo de la losa de transición producida por asiento del terraplén, trabajando la losa con una luz igual al $80 \%$ de su longitud. En la Tabla 3 se muestran las reacciones del tablero del estribo de referencia.
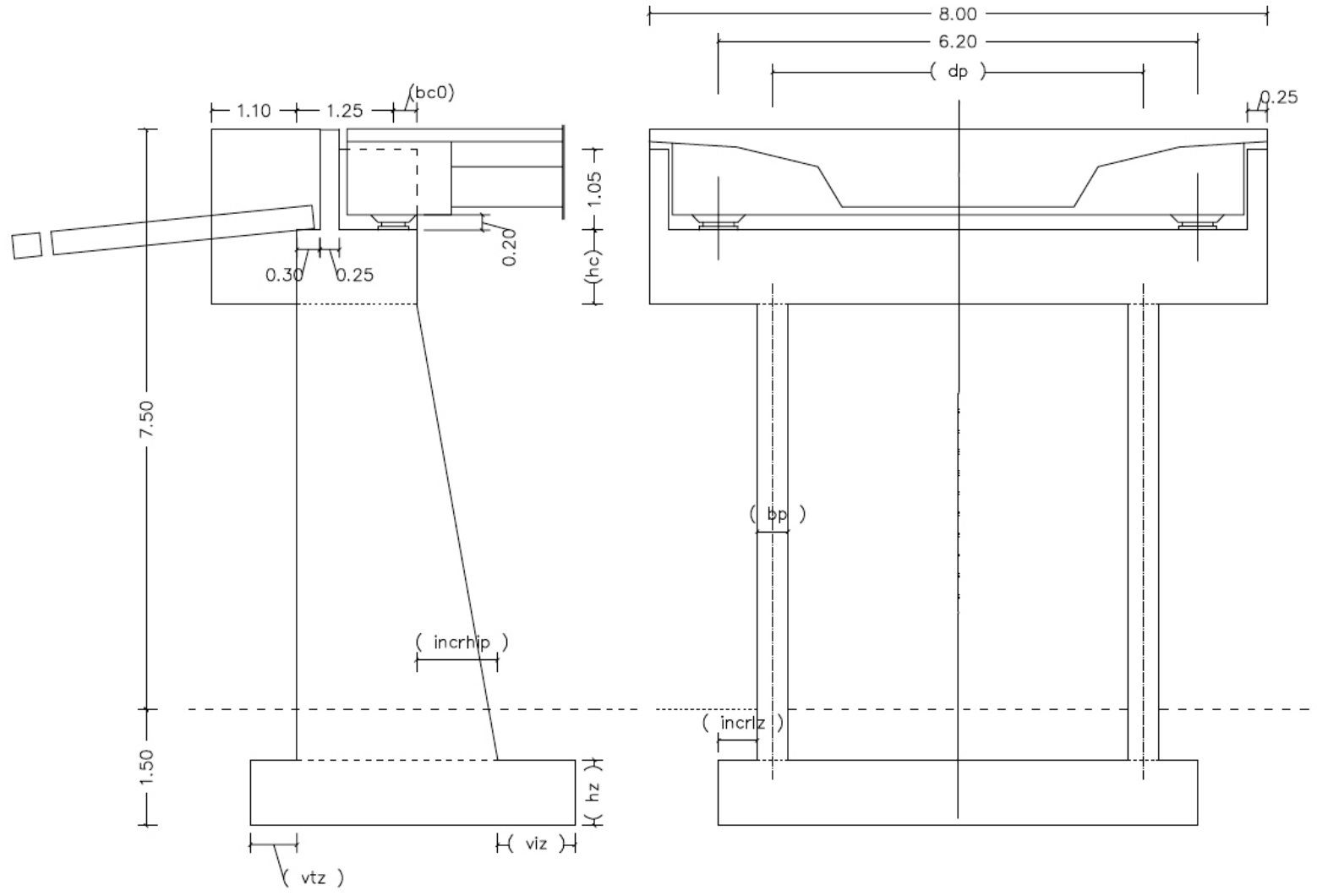

Figura 2. Geometría del estribo abierto: variables y principales parámetros.

Tabla 3. Parámetros de cargas.

\begin{tabular}{|l|c|}
\hline \multicolumn{1}{|c|}{ Descripción } & Valor \\
\hline Reacciones verticales (por apoyo): & $380,00 \mathrm{kN}$ \\
\hline - debida al peso propio y cargas muertas & $280,00 \mathrm{kN}$ \\
\hline - hiperestática del pretensado & $518,25 \mathrm{kN}$ \\
\hline - máxima debida a sobrecarga de tráfico simétrica & $-64,90 \mathrm{kN}$ \\
\hline - mínima debida a sobrecarga de tráfico simétrica & $734,10 \mathrm{kN}$ \\
\hline - máxima debida a sobrecarga de tráfico excéntrica de máximo torsor & $102,02 \mathrm{kN}$ \\
\hline - mínima debida a sobrecarga de tráfico excéntrica de máximo torsor & \\
\hline Reacciones horizontales longitudinales (suma de las dos) & $55,05 \mathrm{kN}$ \\
\hline - debidas a frenado de la sobrecarga de tráfico & $157,90 \mathrm{kN}$ \\
\hline - debidas a deformación & 0,10 \\
\hline - tanto por uno de la reacción de deformación que se considera en sentido contrario & $39,75 \mathrm{kN}$ \\
\hline $\begin{array}{l}\text { Reacciones horizontales transversales (suma de las dos) debidas al viento (dividida por } 2 \text { por simultaneidad con el } \\
\text { tráfico según IAP) actuando en cualquier sentido }\end{array}$ & $10,00 \mathrm{kN} / \mathrm{m}^{2}$ \\
\hline Sobrecarga de tráfico sobre el terraplén & \\
\hline
\end{tabular}



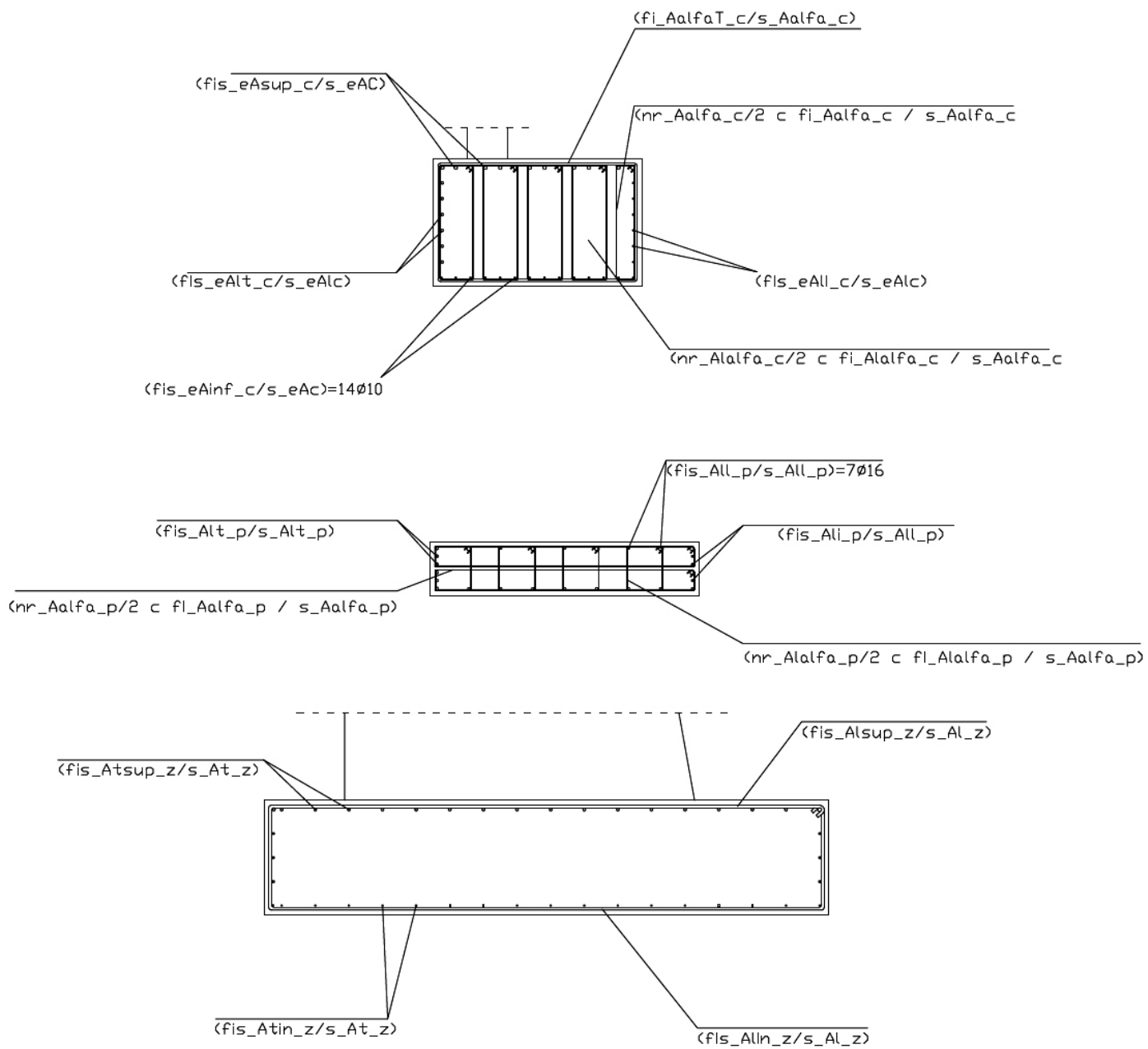

Figura 3. Variables de armado. Secciones del cabezal, pilares y zapata, respectivamente.

El cálculo en el plano longitudinal del puente considera el cabezal ante cargas horizontales como una viga isostática apoyada en los fustes, que se contemplan como ménsulas empotradas en la zapata. Para el cálculo de esfuerzos en el plano transversal, el estribo se modeliza como un pórtico biempotrado en su base. La zapata se considera una cimentación rígida con reparto lineal de tensiones sobre el terreno. La envolvente de los esfuerzos contempla 145 combinaciones para el cabezal y los pilares, y 12 más para la zapata, realizándose las comprobaciones según la norma EHE (26).

\section{ALGORITMOS HÍBRIDOS DE ESCALADA ESTOCÁSTICA EMPLEADOS}

La búsqueda local empleada constituye una técnica iterativa que, partiendo de una solución arbitraria, intenta mejorarla realizando pequeños movimientos en la solución original. Si se cambia la solución cuando mejora la función objetivo, la solución final queda frecuentemente atrapada en óptimos locales de baja calidad. Para superar esta debilidad se puede admitir un empeoramiento estratégico de la solución actual.

Por otra parte, la hibridación de las metaheurísticas explota el carácter complementario de diversas estrategias (10). En este trabajo se plantea la hibridación de técnicas de escalada estocástica como el recocido simulado ( $S A$, simulated annealing) o la aceptación por umbrales (TA, threshold accepting) con un movimiento de búsqueda local, basado en la mutación de los algoritmos genéticos (GA, genetic algorithm). Algunos investigadores (27) (28) han implementado estrategias híbridas que complementan los efectos sinérgicos de GA y SA. De hecho, la técnica se ha empleado en la optimización de tableros con vigas prefabricadas (29), mientras que aquí se propone una hibridación similar con TA. El movimiento local se basa en la mutación típica de los GA. Se ensayaron mutaciones con el 10, 20, 30, 40, 60, 80 y $100 \%$ de las variables, de forma que el mejor movimiento encontrado, dentro de una búsqueda local de máximo gradiente, fue la modificación de hasta el $20 \%$ de las variables.

\subsection{Recocido simulado híbrido con operador de mutación (SAMO)}

En este trabajo se ha empleado una variante híbrida del SA denominada SAMO (hybrid simulated annealing algorithm with a mutation operator) (29). SA se basa en la cristalización de los metales. En efecto, el metal tiende a adoptar una estructura cristalina correspondiente con un estado termodinámico de mínima energía si se calienta suficientemente y desciende la temperatura de forma lenta y controlada. El criterio de aceptación de una nueva solución lo gobierna la 
expresión de Bolzman $\exp (-\Delta E / T)$, donde $\Delta \mathrm{E}$ es el incremento del coste y $T$ es un parámetro denominado temperatura. El algoritmo comienza con una solución aleatoria y con una temperatura inicial elevada. Esta solución se modifica con un movimiento aleatorio de parte de sus variables y se acepta siempre que mejore a la solución previa. Pero también se acepta una solución de mayor coste cuando un número aleatorio entre o y 1 es menor a la expresión $\exp (-\Delta E / T)$. La probabilidad de aceptar soluciones peores disminuye con la temperatura. Dicha reducción suele realizarse geométricamente $\left(T^{\prime}=k T\right)$ por medio de un coeficiente de enfriamiento $k$, manteniéndose constante en un número de iteraciones denominado «cadena de Markov». El algoritmo se detiene cuando la temperatura llega a un porcentaje pequeño de la temperatura inicial o bien no existen mejoras en un número consecutivo de cadenas. En ocasiones se permite un recalentamiento al final del proceso.

En la Figura 4 se ha representado el diagrama de flujo correspondiente a este algoritmo híbrido.

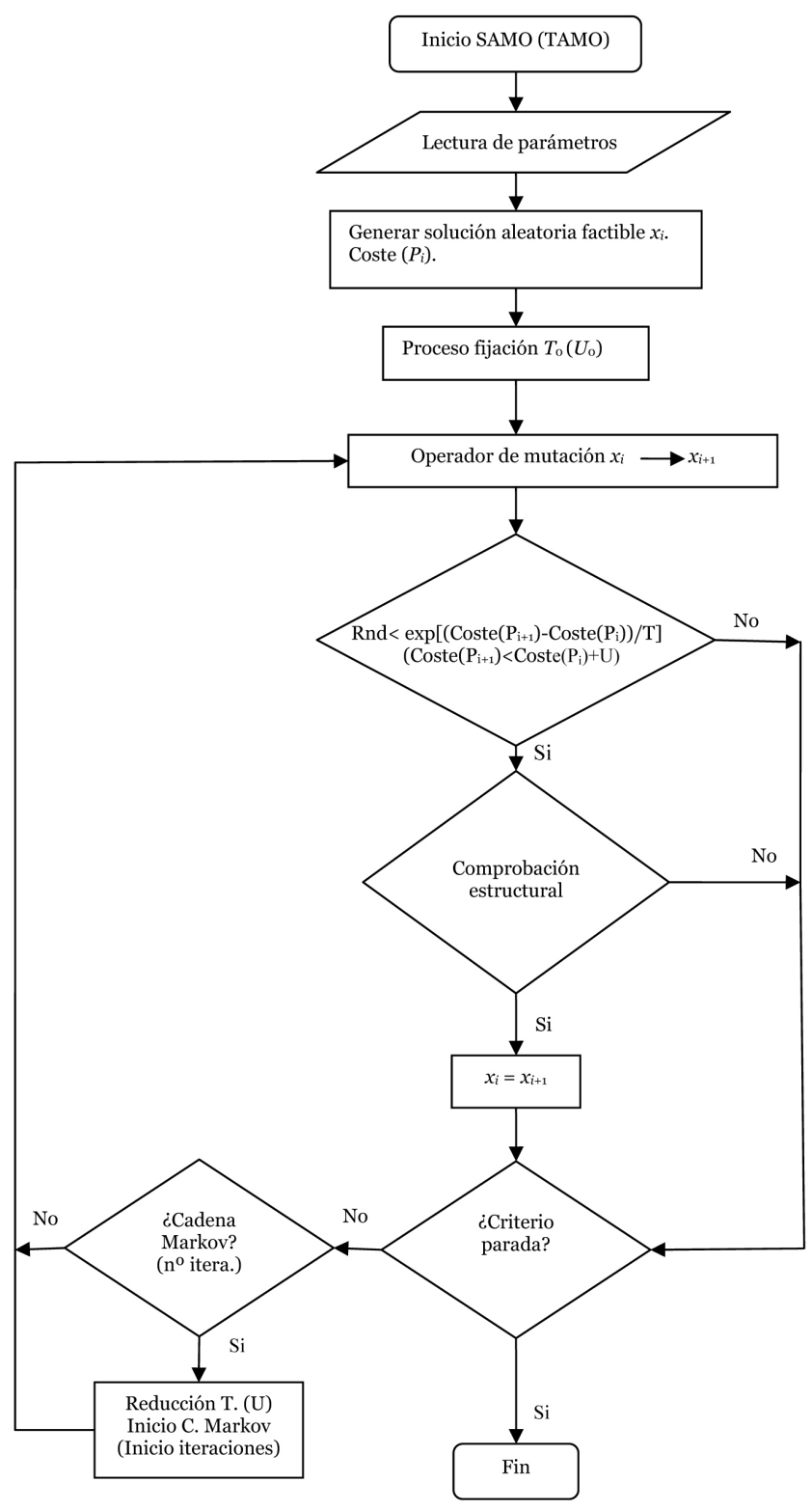

Figura 4. Diagrama de flujo de los algoritmos SAMO (TAMO).
SAMO requiere la calibración de la temperatura inicial, de la longitud de las cadenas de Markov y del coeficiente de enfriamiento. La temperatura inicial se ajusta siguiendo el método de Medina (30), que consiste en elegir una temperatura inicial arbitraria y comprobar, durante una cadena, que el número de aceptaciones se encuentran entre el 20 y $40 \%$. Si el porcentaje es superior al $40 \%$, se divide la temperatura; si no llega al $20 \%$, se duplica. Como primer tanteo de la temperatura $T_{o}$ se ha tomado el equivalente al 2,5\% del coste de una solución media inicial. La calibración de los parámetros se realizó repitiendo cada combinación 9 veces debido al carácter estocástico del algoritmo, resultando una longitud de cadena de 10.000 y un coeficiente de enfriamiento $k=0,80$ (Figura 5). Como criterio de parada se establece el cumplimiento simultáneo de una temperatura menor del $2 \%$ de la inicial $T_{o}$ y del agotamiento de la cadena de Markov sin haber mejorado la solución actual. Para mejorar la solución encontrada, se permiten recalentamientos (31). Así, el algoritmo vuelve a iniciarse con una temperatura que es la mayor de la mitad de la temperatura inicial del proceso anterior o de aquella para la que se encontró la mejor solución, continuando hasta que no existen mejoras (Figura 6).

\subsection{Aceptación por umbrales híbrido con operador de mutación (TAMO)}

En este trabajo se presenta un nuevo algoritmo, TA hibridado con un operador de mutación que se ha denominado TAMO (hybrid threshold accepting algorithm with a mutation operator). El método constituye una variante de TA que sigue una hibridación similar a SAMO. TA es un algoritmo de escalada (32) (33) que permite la degradación de una solución siempre que no sobrepase un umbral determinado. El algoritmo comienza con una solución aleatoria y con un umbral inicial. La solución se modifica mediante una mutación aleatoria. Se evalúa la nueva solución y se acepta la nueva solución si mejora la función objetivo o bien el empeoramiento se acota con el umbral previsto. De forma análoga a SA, el umbral se mantiene constante en un ciclo de movimientos, tras el cual disminuye al multiplicarlo por un coeficiente $k$ menor a la unidad. El algoritmo finaliza cuando el umbral llega a un pequeño porcentaje del valor inicial. En la Figura 4 se representa el diagrama de flujo de TAMO.

El método requiere una calibración del umbral inicial, del número de iteraciones de cada ciclo y del coeficiente reductor. Siguiendo un procedimiento similar al SAMO, el umbral inicial se determina con el procedimiento de Medina (30). La calibración de los parámetros conduce a una longitud de 2.000 iteraciones por ciclo y un coeficiente $k=0,90$ de disminución del umbral. Se utiliza como criterio de parada el empleado en SAMO. Asimismo, se permiten reinicios sucesivos con umbrales iniciales decrecientes hasta no encontrar mejoras. Análogamente, el nuevo umbral es el mayor de los valores formados por la mitad del umbral inicial del proceso anterior o el umbral con el que se encontró la mejor solución.

\section{ESTUDIO PARAMÉTRICO Y ANÁLISIS DE RESULTADOS}

Los programas se han desarrollado íntegramente en FORTRAN utilizando un ordenador personal con procesador Intel Core i7-3820 CPU @ 3,50 GHz y con 16,o GB de memoria RAM y sistema operativo de 64 bits. Tras la calibración de SAMO y TAMO, los costes mínimos encontrados para el estribo de re- 


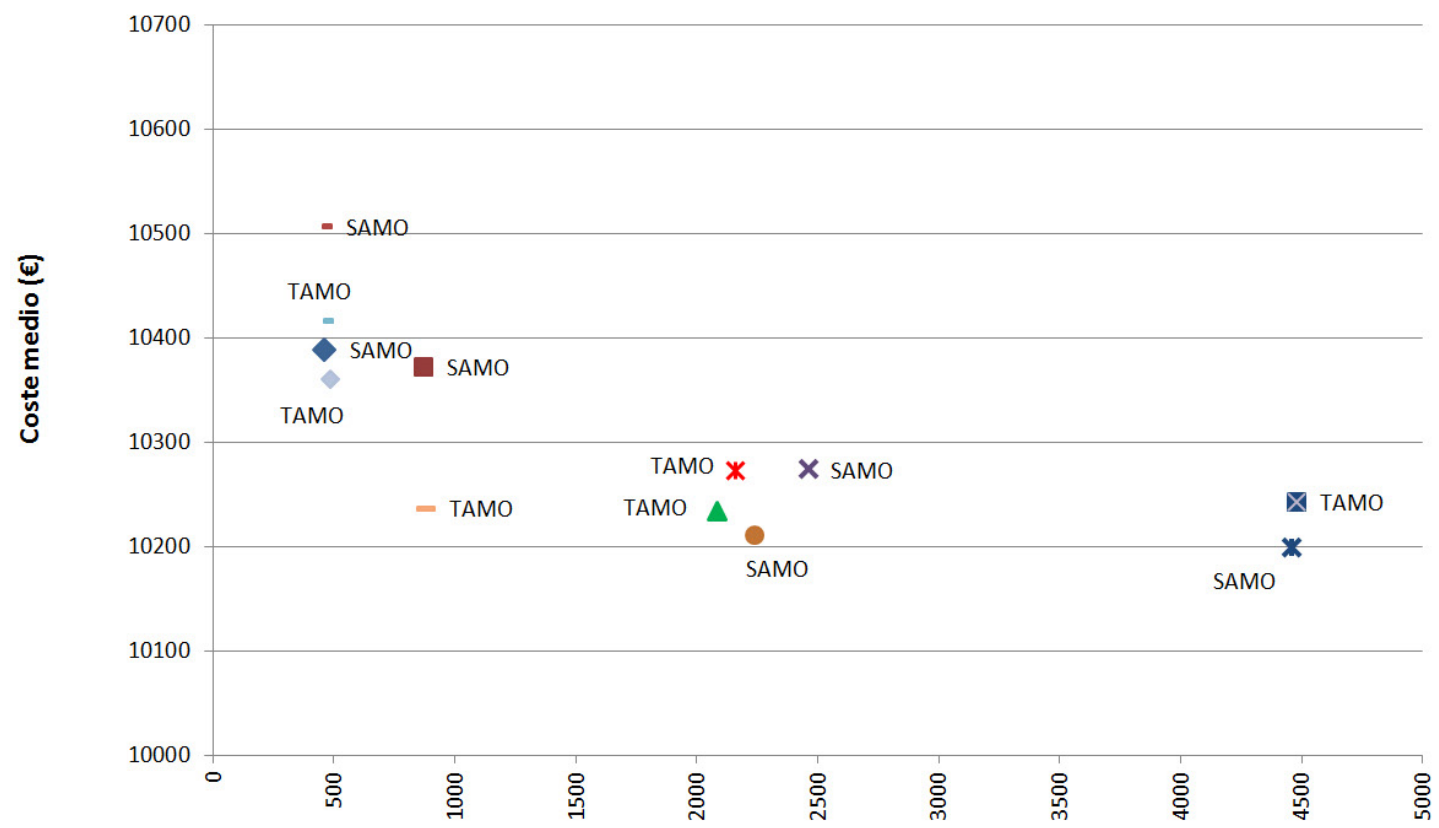

- SAMO/5000/0.80

-SAMO $/ 5000 / 0.85$

- SAMO/5000/0.90

- SAMO $/ 10000 / 0.80$

XSAMO/10000/0.85

×SAMO $/ 10000 / 0.90$

- TAMO/5000/0.80

$\bullet$ TAMO $/ 5000 / 0.85$

- TAMO $/ 5000 / 0.90$

$\triangle \mathrm{TAMO} / 10000 / 0.80$

*TAMO $/ 10000 / 0.85$

ब TAMO $/ 10000 / 0.90$

Figura 5. Comparativa de las mejores soluciones SAMO/TAMO.

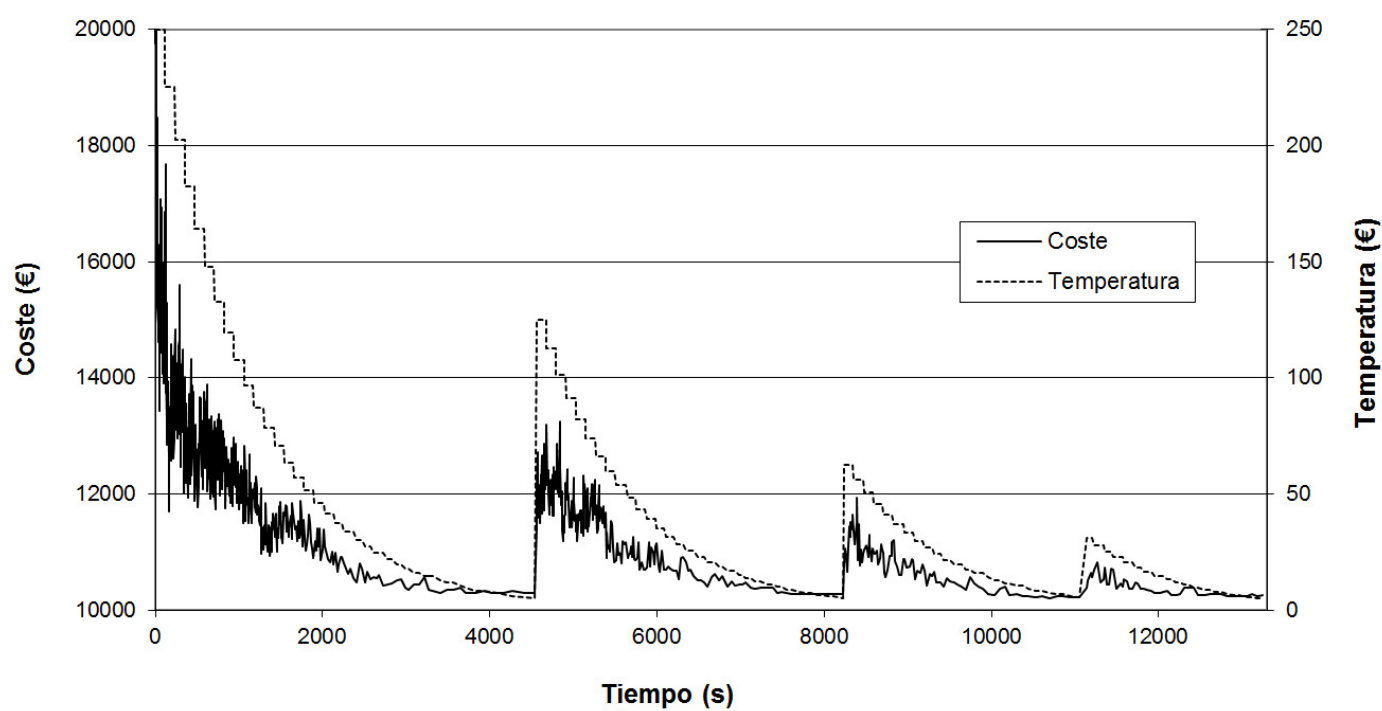

Figura 6. Evolución del SAMO con recalentamientos. Longitud cadena de Markov 10000, $k=0,90$.

ferencia fueron de 10.132,19 $€$ y 10.211,78 $€$, respectivamente. Esto significa que la mejor solución encontrada, con SAMO, mejora un 18,77 \% el coste de la referencia. En ambos casos se ejecutaron los algoritmos 9 veces, comprobándose desviaciones de los valores medios respecto al mínimo muy pequeños, entre un 0,5 y un 2,2\%. Los tiempos de cálculo medios han sido de 34 minutos en el caso de SAMO y de 33 minutos en el caso de TAMO. En la Tabla 4 se aprecian las diferencias existentes entre el estribo de menor coste y el de referencia, mientras que la Tabla 5 detalla la composición de los ahorros obtenidos.

A continuación se estudia la influencia de la altura total del estribo y de las tensiones admisibles del terreno. Se han analizado 6, 9, 12 y $15 \mathrm{~m}$ de altura y unas tensiones entre 0,20 y 0,50 MPa, en escalones de 0,10 MPa. Son 15 combinaciones, puesto que para alturas de $15 \mathrm{~m}$ y tensiones de o,20 $\mathrm{MPa}$, no se dan soluciones factibles. Los resultados de coste mínimo se han representado en la Figura 7 y sus principales características en la Tabla 6, incluyendo las cuantías de armado del cabezal $(\omega c)$, pilares $(\omega \mathrm{p})$ y zapata $(\omega z)$. La Figura 7 indica un incremento del coste, tanto cuando aumenta la altura del estribo, como cuando disminuye la tensión admisible del terreno.

El hormigón empleado en la zapata es el de menor resistencia característica, $25 \mathrm{MPa}$, independientemente de la altura y del terreno, coincidiendo con la empleada en el estribo de referencia (Tablas 4 y 6). Sin embargo, el hormigón del alzado a partir de $12 \mathrm{~m}$ de altura es, en ocasiones, de una resistencia algo mayor, de $30 \mathrm{MPa}$. 
Tabla 4. Estribo de referencia (E.R.) vs. Estribo optimizado (E.O.).

\begin{tabular}{|c|c|c|}
\hline \multirow{2}{*}{$\begin{array}{c}\text { Altura (m) } \\
\text { Diseño }\end{array}$} & \multicolumn{2}{|c|}{9} \\
\hline & E.R. & E.O. \\
\hline Coste $(€)$ & 12475 & 10132 \\
\hline bco & 0,75 & 0,30 \\
\hline$b c$ & 2,00 & 1,55 \\
\hline$h c$ & 0,80 & 0,95 \\
\hline$b p$ & 0,50 & 0,40 \\
\hline$d p$ & 4,70 & 4,80 \\
\hline incrhip & 0,00 & 1,05 \\
\hline hip & 2,00 & 2,60 \\
\hline$h p$ & 5,90 & 5,90 \\
\hline$v t z$ & 1,75 & 0,60 \\
\hline viz & 1,25 & 1,00 \\
\hline$b z$ & 5,00 & 4,20 \\
\hline$h z$ & 1,00 & 0,85 \\
\hline incrlz & 0,65 & 0,50 \\
\hline$l z$ & 6,50 & 6,20 \\
\hline$f c k \_e$ & 30 & 25 \\
\hline$f c k \_z$ & 25 & 25 \\
\hline $3^{*} \mathrm{He} / \mathrm{hip}$ & 13,50 & 10,38 \\
\hline$h p / b p$ & 11,80 & 14,75 \\
\hline$b z / H e$ & 0,56 & 0,47 \\
\hline viz/He & 0,14 & 0,11 \\
\hline$h z / v i z$ & 0,80 & 0,85 \\
\hline$($ incrlz+0.5bp)/dp & 0,19 & 0,15 \\
\hline$(d p-b p) / h z$ & 4,20 & 5,18 \\
\hline$\omega c\left(\mathrm{~kg} / \mathrm{m}^{3}\right)$ & 107,5 & 116,45 \\
\hline$\omega p\left(\mathrm{~kg} / \mathrm{m}^{3}\right)$ & 123,6 & 103, \\
\hline$\omega z\left(\mathrm{~kg} / \mathrm{m}^{3}\right)$ & 78,3 & 37,9 \\
\hline
\end{tabular}

A continuación se comparan los criterios de diseño obtenidos en los estribos optimizados respecto al estribo de referencia y con respecto a las recomendaciones de Arenas y Aparicio (24). El canto de los pilares en la referencia es constante (incrhip $=0$ ), lo cual se corresponde con alturas inferiores a 6 m (21). Sin embargo, los estribos de coste mínimo muestran variabilidad en el canto de los pilares, minimizando el canto superior del pilar, con valores comprendidos entre 1,55 y $1,70 \mathrm{~m}$. Sólo en algunos casos correspondientes a alturas $H e=6 \mathrm{~m}$, se ha mantenido el canto constante, con unas alturas de pilares de unos $3 \mathrm{~m}$ (ver Tabla 6).

La relación encontrada entre el canto del cabezal y la distancia entre pilares $(h c / d p)$, oscila entre $1 / 5$ y $1 / 7,6$ en los estribos optimizados, similar a la empleada en la referencia, de 1/5,9. Esta relación disminuye al aumentar la altura del estribo y al reducirse la tensión admisible, puesto que la mayor superficie de zapata permite distanciar los pilares. Los resultados en los estribos de coste mínimo son similares a las recomendaciones de Arenas y Aparicio (24), con valores comprendidos entre 1/4,4 y $1 / 5,0$.

Otra relación de interés es la distancia de la reacción al paramento del pilar respecto al canto útil del cabezal. Las recomendaciones (24) indican ratios inferiores a 0,75 para no armar a cortante, lo cual se cumple en la referencia. En los estribos optimizados estos valores son más bajos, entre 0,07 y 0,55 , salvo para los de menor altura entre 0,20 y 1,70, lo cual muestra la eficiencia del algoritmo para evitar dicha armadura de cortante. La relación encontrada en los estribos optimizados entre el canto inferior del pilar divido por 3 -por ser el empuje real 3 veces el ancho del pilar- y la altura del estribo (hip/3He) presenta valores medios de 1/9,8, dentro del rango recomendado (21), entre $1 / 8$ y $1 / 12$, mientras que el ratio de la referencia es de $1 / 13,5$.

Un caso frecuente en los pasos superiores son alturas de estribo $\mathrm{He}=9 \mathrm{~m}$. En los estribos de coste mínimo la relación entre el ancho y la altura del pilar $(b p / h p)$ oscila entre 1/16,6 y $1 / 14,8$, situándose los valores de $b p$ entre 0,35 y 0,40 m. Este criterio encaja con la recomendación (24) de que la relación sea del orden de 1/15, evitando pilares inferiores a o,40 m para evitar el pandeo transversal. Esta relación es mayor para la referencia, 1/11,80, y también aumenta para

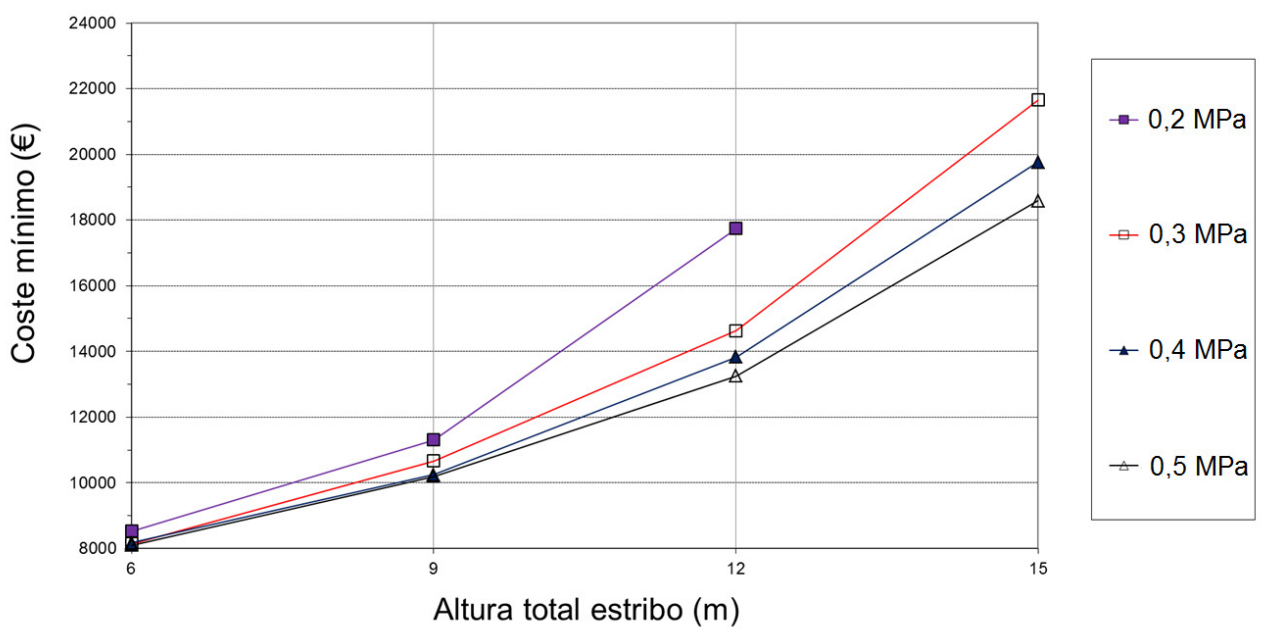

Figura 7. Variación del coste de las mejores soluciones cuando varía la altura total del estribo. 
las estructuras optimizadas de alturas menores a $9 \mathrm{~m}$, con ratios en torno a $1 / 8$ y anchos de pila entre 0,30 y $0,35 \mathrm{~m}$, con cantos constantes o poco variables. Para estribos optimizados de altura mayor a $9 \mathrm{~m}$, la relación disminuye hasta $1 / 30$, con anchuras de pila comprendidas entre 0,40 y $0,45 \mathrm{~m}$.

Los valores recomendados (24) para la relación entre el ancho de la zapata $b z$ y la altura del estribo $\mathrm{He}$ en estribos cerrados, varían entre 0,4 y 0,7, lo que ajusta bien para los estribos optimizados de $9 \mathrm{~m}$ de altura, con relaciones en torno a 0,46 , que compensan el menor empuje de las tierras con su mayor peso. Esta relación llega a 0,37 en el caso de estribos de $15 \mathrm{~m}$ y $0,50 \mathrm{MPa}$ de tensión admisible del terreno y sube a 0,70 cuando la altura es de $6 \mathrm{~m}$ y la tensión de o,20 MPa. Para la referencia, la relación aumenta a 0,56 , lo que explica en gran parte ahorros medios de la estructura optimizada del $18 \%$.

De forma similar, las recomendaciones (24) del ratio entre la puntera y la altura (viz/He) oscilan para estribos cerrados entre 0,15 y 0,25, ajustándose bien, pero los valores de los estribos optimizados se reducen hasta 0,07 con $15 \mathrm{~m}$ de altura; y aumentan en el caso de alturas bajas hasta 0,32 con una altura de $6 \mathrm{~m}$. En la referencia dicha relación es de 0,14. El cociente entre el canto de la zapata $h z$ y la puntera viz para los

Tabla 5. Costes y ahorros entre estribo de referencia y estribo optimizado.

\begin{tabular}{|c|c|c|c|c|c|c|}
\hline \multirow{2}{*}{ Elemento } & \multicolumn{2}{|c|}{ E.R. } & \multicolumn{2}{|c|}{ E.O. } & \multicolumn{2}{|c|}{ Ahorro } \\
\hline & Coste $(\boldsymbol{\epsilon})$ & \% Coste & Coste $(\boldsymbol{\epsilon})$ & \% Coste & (€) & $\%$ \\
\hline Estribo & $12.474,95$ & 100,00 & $10.132,19$ & 100,00 & $2.342,76$ & 100,00 \\
\hline Cabezal & $2.145,11$ & 17,20 & $1.989,68$ & 19,64 & 155,43 & 6,63 \\
\hline Pilares & $2.823,23$ & 22,63 & $2.656,36$ & 26,22 & 166,87 & 7,12 \\
\hline Zapata & $4.582,78$ & 36,74 & $2.638,14$ & 26,04 & $1.944,64$ & 83,01 \\
\hline
\end{tabular}

Tabla 6. Estudio paramétrico de los estribos optimizados para el caso de un puente continuo de dos vanos y $25 \mathrm{~m}$ de luz por vano.

\begin{tabular}{|c|c|c|c|c|c|c|c|c|c|c|c|c|c|c|c|}
\hline Altura (m) & & 6 & & & & & 9 & & & 1 & & & & 15 & \\
\hline Tensión (10 $\left.{ }^{-1} \mathrm{Mpa}\right)$ & 2 & 3 & 4 & 5 & 2 & 3 & 4 & 5 & 2 & 3 & 4 & 5 & 3 & 4 & 5 \\
\hline Coste $(€)$ & 8532 & 8139 & 8175 & 8099 & 11302 & 10663 & 10250 & 10200 & 17737 & 14632 & 13833 & 13254 & 21654 & 19760 & 18581 \\
\hline bco & 0,55 & 0,50 & 0,80 & 0,70 & 0,35 & 0,30 & 0,40 & 0,35 & 0,30 & 0,45 & 0,35 & 0,30 & 0,45 & 0,30 & 0,30 \\
\hline$b c$ & 1,80 & 1,75 & 2,05 & 1,95 & 1,60 & 1,55 & 1,65 & 1,60 & 1,55 & 1,70 & 1,60 & 1,55 & 1,70 & 1,55 & 1,55 \\
\hline$h c$ & 1,05 & 0,90 & 0,55 & 0,75 & 0,75 & 0,85 & 0,80 & 0,85 & 0,85 & 0,75 & 0,85 & 0,85 & 0,75 & 0,95 & 0,80 \\
\hline$b p$ & 0,30 & 0,35 & 0,30 & 0,35 & 0,40 & 0,40 & 0,35 & 0,35 & 0,40 & 0,40 & 0,40 & 0,40 & 0,40 & 0,45 & 0,40 \\
\hline$d p$ & 3,75 & 4,70 & 5,70 & 4,85 & 5,55 & 5,20 & 5,15 & 5,20 & 5,55 & 5,70 & 5,65 & 5,45 & 5,45 & 5,45 & 5,70 \\
\hline incrhip & 0,70 & 0,00 & 0,00 & 0,00 & 1,25 & 1,25 & 0,75 & 0,75 & 4,85 & 1,85 & 1,90 & 1,70 & 2,65 & 2,70 & 2,20 \\
\hline hip & 2,50 & 1,75 & 2,05 & 1,95 & 2,85 & 2,80 & 2,40 & 2,35 & 6,40 & 3,55 & 3,50 & 3,25 & 4,35 & 4,25 & 3,75 \\
\hline$h p$ & 2,85 & 2,75 & 2,95 & 2,90 & 6,15 & 6,05 & 5,85 & 5,80 & 9,35 & 9,15 & 9,10 & 9,05 & 11,95 & 11,90 & 11,90 \\
\hline$v t z$ & 0,20 & 0,00 & 0,00 & 0,00 & 0,00 & 0,10 & 0,00 & 0,10 & 0,60 & 0,60 & 0,50 & 0,60 & 1,10 & 0,80 & 0,70 \\
\hline viz & 1,50 & 1,90 & 1,20 & 1,10 & 2,50 & 1,40 & 1,70 & 1,60 & 0,90 & 1,80 & 0,90 & 0,90 & 2,70 & 1,00 & 1,10 \\
\hline$b z$ & 4,20 & 3,65 & 3,25 & 3,05 & 5,35 & 4,30 & 4,10 & 4,05 & 7,90 & 5,95 & 4,90 & 4,75 & 8,15 & 6,05 & 5,55 \\
\hline$h z$ & 0,80 & 1,05 & 1,20 & 1,05 & 0,80 & 0,80 & 1,05 & 1,05 & 0,50 & 0,80 & 0,75 & 0,80 & 1,00 & 0,85 & 1,00 \\
\hline incrlz & 0,30 & 0,00 & 0,00 & 0,30 & 0,50 & 0,80 & 0,00 & 0,00 & 2,00 & 0,80 & 0,90 & 0,70 & 1,00 & 2,00 & 0,90 \\
\hline$l z$ & 4,65 & 5,05 & 6,00 & 5,80 & 6,95 & 7,20 & 5,50 & 5,55 & 9,95 & 7,70 & 7,85 & 7,25 & 7,85 & 9,90 & 7,90 \\
\hline$f c k \_e$ & 25 & 25 & 25 & 25 & 25 & 25 & 25 & 25 & 30 & 25 & 25 & 25 & 30 & 30 & 25 \\
\hline$f c k \_z$ & 25 & 25 & 25 & 25 & 25 & 25 & 25 & 25 & 25 & 25 & 25 & 25 & 25 & 25 & 25 \\
\hline $3^{*} \mathrm{He} / \mathrm{hip}$ & 7,2 & 10,29 & 8,78 & 9,23 & 9,47 & 9,64 & 11,25 & 11,49 & 5,63 & 10,14 & 10,29 & 11,08 & 10,34 & 10,59 & 12,00 \\
\hline$h p / b p$ & 9,5 & 7,86 & 9,83 & 8,29 & 15,38 & 15,13 & 16,71 & 16,57 & 23,38 & 22,88 & 22,75 & 22,63 & 29,88 & 26,44 & 29,75 \\
\hline$b z / H e$ & 0,70 & 0,61 & 0,54 & 0,51 & 0,59 & 0,48 & 0,46 & 0,45 & 0,66 & 0,50 & 0,41 & 0,40 & 0,54 & 0,40 & 0,37 \\
\hline viz/He & 0,25 & 0,32 & 0,20 & 0,18 & 0,28 & 0,16 & 0,19 & 0,18 & 0,08 & 0,15 & 0,08 & 0,08 & 0,18 & 0,07 & 0,07 \\
\hline$h z / v i z$ & 0,53 & 0,55 & 1,00 & 0,95 & 0,32 & 0,57 & 0,62 & 0,66 & 0,56 & 0,44 & 0,83 & 0,89 & 0,37 & 0,85 & 0,91 \\
\hline$($ incrlz $+0.5 b p) / d p$ & 0,12 & 0,04 & 0,03 & 0,10 & 0,13 & 0,19 & 0,03 & 0,03 & 0,40 & 0,18 & 0,19 & 0,17 & 0,22 & 0,41 & 0,19 \\
\hline$(d p-b p) / h z$ & 4,31 & 4,14 & 4,50 & 4,29 & 6,44 & 6,00 & 4,57 & 4,62 & 10,30 & 6,63 & 7,00 & 6,31 & 5,05 & 5,88 & 5,30 \\
\hline$\omega c\left(\mathrm{~kg} / \mathrm{m}^{3}\right)$ & 103 & 101 & 130 & 104 & 140 & 132 & 129 & 124 & 129 & 125 & 117 & 128 & 133 & 106 & 136 \\
\hline$\omega p\left(\mathrm{~kg} / \mathrm{m}^{3}\right)$ & 131 & 125 & 103 & 117 & 91 & 110 & 148 & 156 & 94 & 129 & 152 & 149 & 183 & 151 & 217 \\
\hline$\omega z\left(\mathrm{~kg} / \mathrm{m}^{3}\right)$ & 45 & 45 & 41 & 47 & 54 & 42 & 45 & 44 & 48 & 51 & 42 & 41 & 44 & 35 & 39 \\
\hline
\end{tabular}


estribos optimizados toma valores entre 0,32 y 1,00, aumentando para cada altura con la tensión admisible del terreno. El valor medio es igual a 0,67, por debajo de la propuesta (24) para estribos cerrados de 0,70 , mientras que para la referencia es de 0,80 .

Sólo para los estribos más altos, de 12 y 15 m, con tensiones admisibles iguales o cercanas a o,2 $\mathrm{MPa}$, las zapatas quedan compensadas a flexión transversal, con ratios entre los voladizos de la zapata (incrlz $+0.5 b p$ ) y la distancia entre pilares $d p$ en torno a o,40. Para el resto de casos, las relaciones oscilan entre 0,00 y 0,20. En la referencia, se supera ligeramente el valor de 0,19. Ello indica que con zapatas aisladas se reduciría el coste de la cimentación y del cabezal al permitir fustes justo bajo los apoyos y eliminar los esfuerzos en el cabezal producidos por las reacciones. Este criterio no se corresponde con la práctica usual para los estribos y terrenos habituales ni con las recomendaciones (24).

Otro aspecto importante es la relación entre la distancia entre caras de pilares $(d p-b p)$ y el canto de la zapata $(h z)$. Según la EHE (26), dicha relación debe ser superior a 4 para una cimentación flexible, cumpliéndose en todos los casos para los estribos optimizados, aunque con esbelteces pequeñas, pues la relación media es de 5,70. Para alturas de $9 \mathrm{~m}$ dicho valor de 4,90 es más esbelto que los 4,20 del estribo de referencia.

A continuación se analizan las comprobaciones críticas y las disposiciones de armado. En el cabezal es necesario comprobar la flexión transversal -flexión esviada- y el cortante lateral debido al empuje de tierras sobre el mismo y las aletas. Se hace notar que esta condición no se cumple en la referencia. Estas comprobaciones, junto con el torsor también en voladizo y el cortante vertical en apoyo, han sido las más críticas en los estribos de coste mínimo. Sin embargo, en las estructuras optimizadas no son críticos los esfuerzos combinados, en línea con las recomendaciones (24), que no los considera. En las soluciones optimizadas, los cercos de cortante son distintos de los de torsión, sirviendo para atar las armaduras en compresión, al igual que ocurre con la referencia, pero no siguiendo lo recomendado por Arenas y Aparicio (24).

La comprobación de la flexión compuesta esviada con pandeo transversal no se cumple en los pilares de la referencia, siendo la condición más crítica tanto en la sección superior e inferior de los estribos optimizados. Es necesario atar las armaduras longitudinales con cercos en las dos direcciones debido a la flexión compuesta, cosa que no ocurre con la referencia ni en los criterios de Arenas y Aparicio (24), y es suficiente una sola capa de armadura longitudinal en el trasdós. No son críticos el cortante ni la fisuración. En la zapata las comprobaciones críticas son: la plastificación para las reacciones torsoras, el vuelco y el cortante, junto con las de flexión longitudinal en puntera y transversal en centro vano. En los estribos más altos y con menor tensión admisible -zapata más esbelta-, el vuelco deja de ser crítico y el cortante es más desfavorable en el apoyo (cortante transversal) que en la puntera. En la referencia, el cortante en el talón es más desfavorable que el cortante transversal en apoyo, dado que es necesario un talón apreciable.

Por último, en la Tabla 5 se desglosan los ahorros del estribo de referencia respecto a la de coste mínimo, con un valor total de $2341,76 €$, lo cual supone un ahorro del 18,77\%. El 83,0 \% del ahorro se debe a la zapata, no sólo se disminuye el volumen de 32,50 a $22,13 \mathrm{~m}^{3}$, sino que el acero baja de 2524,24 a $838,84 \mathrm{~kg}$. Además, en la Figura 7 se observa que, para un mismo terreno, aumenta fuertemente el coste con la altura del estribo. Por otro lado, cuando aumenta la tensión admisible a igualdad de altura del estribo, los costes son menores al reducirse la magnitud de la zapata. Cuando el estribo es bajo la repercusión de la zapata es pequeña en el conjunto.

\section{CONCLUSIONES}

Se han aplicado metaheurísticas híbridas de escalada estocástica que han mostrado su eficiencia en el diseño automático de coste mínimo de estribos abiertos de puentes de carreteras. Se han conseguido ahorros significativos, del 18,77\% cuando se compara con un estribo de referencia. En el trabajo se ha presentado un nuevo algoritmo híbrido, TAMO, que presenta diferencias poco significativas en cuanto a la calidad de las soluciones obtenidas respecto al algoritmo SAMO, inferiores al o,5 \% a favor de SAMO.

Respecto al diseño estructural, el estudio paramétrico realizado para distintas alturas y tensiones admisibles del terreno, ofrece criterios de predimensionamiento. Además, los estribos de coste mínimo se comportan del siguiente modo:

a) La resistencia característica del hormigón empleado en cualquier parte del estribo es la menor disponible, con disposiciones de pilares de canto variable, lo cual no es habitual en estas estructuras, especialmente con alturas de pilares inferiores a $6 \mathrm{~m}$.

b) El ahorro económico se localiza en la zapata, gracias al notable aumento del canto inferior de los pilares, lo que permite disminuir el talón sin aumentar la puntera.

c) Los pilares son esbeltos en el plano transversal al tablero, con ancho medio de $0,40 \mathrm{~m}$, por lo que es necesario considerar la flexión compuesta esviada con pandeo.

d) En contra del criterio habitual, los estribos más eficientes desde el punto de vista económico son aquellos que presentan zapatas aisladas.

\section{AGRADECIMIENTOS}

Este estudio ha sido financiado por el Ministerio Español de Economía y Competitividad y fondos FEDER (proyecto de investigación BIA2014-56574-R), así como por la Universitat Politècnica de València (proyecto SP20120341). 


\section{REFERENCIAS}

(1) Martí, J.V., Yepes, V., González-Vidosa, F. (2015). A memetic algorithm approach to designing of precast-prestressed concrete road bridges with steel fiber-reinforcement. Journal of Structural Engineering ASCE, 141(2): 04014114, doi: http://doi.org/10.1061/(ASCE)ST.1943-541X.0001058.

(2) Martínez, F.J., González-Vidosa, F., Hospitaler, A., Yepes, V. (2010). Heuristic optimization of RC bridge piers with rectangular hollow sections. Computers \& Structures, 88(5-6): 375-386, doi: http://dx.doi.org/10.1016/j. compstruc.2009.11.009.

(3) Hassanain, M.A., Loov, R.E. (2003). Cost optimization of concrete bridge infrastructure. Canadian Journal of Civil Engineering, 30(5): 841-849, doi: http://dx.doi.org/10.1139/lo3-045.

(4) Hernández, S., Fontán, A.N., Díaz, J., Marcos, D. (2010). VTOP. An improved software for design optimization of prestressed concrete beams. Advances in Engineering Software, 41(3): 415-421, doi: http://dx.doi.org/10.1016/j.advengsoft.2009.03.009.

(5) Arockiasamy, M., Butrieng, N., Sivakumar, M. (2004). State-of-the-art of integral abutment bridges: design and practice. Journal of Bridge Engineering, 9(5): 497-506, doi: http://dx.doi.org/10.1061/(ASCE)10840702(2004)9:5(497).

(6) García-Villena, F. A., Carbonell-Márquez, J. F. (2014). Armado óptimo de secciones de hormigón posteso. Informes de la Construcción, 66(EXTRA-1): mo14, doi: http://dx.doi.org/10.3989/ic.13.099.

(7) Calvo, J., Gracia, J., Bayo, E. (2014). Aplicación web para el análisis y diseño de estructuras. Informes de la Construcción, 66(EXTRA-1): moo1, doi: http://dx.doi.org/10.3989/ic.13.075.

(8) Parras Galán, L., Montes Tubío, M., García Guzmán, A., Entrenas Angulo, J.A., de Dios Palomares, R. (1985). Un método de optimización de estructuras articuladas estáticamente determinadas. Informes de la Construcción, 37(372): 65-73, doi: http://dx.doi.org/10.3989/ic.1985.v37.i372.1857.

(9) Cohn, M.Z., Dinovitzer, A.S. (1994). Application of structural optimization. Journal of Structural Engineering, 120(2): 617-649, doi: http://dx.doi.org/10.1061/(ASCE)0733-9445(1994)120:2(617).

(10) Blum, C., Puchinger, J., Raidl, G.R., Roli, A. (2011). Hybrid metaheuristics in combinatorial optimization: A survey. Applied Soft Computing, 11(6): 4135-4151, doi: http://dx.doi.org/10.1016/j.asoc.2011.02.032.

(11) Holland, J.H. (1975). Adaptation in Natural and Artificial Systems. Ann Arbor, MI: University of Michigan Press.

(12) Kirkpatrick, S., Gelatt, C.D., Vecchi, M.P. (1983). Optimization by simulated annealing. Science, 220(4598): 671-680, doi: http://dx.doi.org/10.1126/science.220.4598.671.

(13) Krishnanand, K.N., Ghose, D. (2009). Glowworm swarm optimisation: a new method for optimising multi-modal functions. International Journal of Computational Intelligence Studies, 1(1): 93-119, doi: http://dx.doi.org/10.1504/ IJCIStudies.2009.02534.

(14) Kicinger, R., Arciszewski, T., de Jong, K. (2005). Evolutionary computation and structural design: A survey of the stateof-the-art. Computers \& Structures, 83(23-24): 1943-1978, doi: http://dx.doi.org/10.1016/j.compstruc.2005.03.002.

(15) Vázquez, M. (1995). Un nuevo algoritmo para la optimización de estructuras: el recocido simulado. Informes de la Construcción, 46(436): 49-69, doi: http://dx.doi.org/10.3989/ic.1995.v46.i436.1084.

(16) Orta, B. (2008). Optimización de láminas reticuladas de una capa. Informes de la Construcción, 60(511): 59-72, doi: http://dx.doi.org/10.3989/ic.2008.v6o.i511.747.

(17) Balling, R.J.; Yao, X. (1997). Optimization of reinforced concrete frames. Journal of Structural Engineering, 123(2): 193-202, doi: http://dx.doi.org/10.1061/(ASCE)0733-9445(1997)123:2(193).

(18) Perea, C., Alcalá, J., Yepes, V., González-Vidosa, F., Hospitaler, A. (2008). Design of reinforced concrete bridge frames by heuristic optimization. Advances in Engineering Software, 39(8): 676-688, doi: http://doi.org/10.1016/j.advengsoft.2007.07.007.

(19) Carbonell, A. González-Vidosa, F. Yepes V. (2011). Design of reinforced concrete road vaults by heuristic optimization. Advances in Engineering Software, 42(4): 151-159, doi: http://doi.org/10.1016/j.advengsoft.2011.01.002.

(20) Yepes, V., Alcalá, J., Perea, C., González-Vidosa, F. (2008). A parametric study of optimum earth-retaining walls by simulated annealing. Engineering Structures, 30(3): 821-830, doi: http://doi.org/10.1016/j.engstruct.2007.05.023.

(21) Yepes, V., Gonzalez-Vidosa, F., Alcalá, J., Villalba, P. (2012). $\mathrm{CO}_{2}$-optimization design of reinforced concrete retaining walls based on a VNS-threshold acceptance strategy. Journal of Computing in Civil Engineering, 26(3): 378-386, doi: http://doi.org/10.1061/(ASCE)CP.1943-5487.0000140.

(22) Yepes, V., Martí, J.V., García-Segura, T. (2015). Cost and $\mathrm{CO}_{2}$ emission optimization of precast-prestressed concrete Ubeam road bridges by a hybrid glowworm swarm algorithm. Automation in Construction, 49: 123-134, doi: http://doi. org/10.1016/j.autcon.2014.10.013.

(23) Martínez-Martín, F., González-Vidosa, F., Hospitaler, A., Yepes, V. (2013). A parametric study of optimum tall piers for railway bridge viaducts. Structural Engineering and Mechanics, 45(6): 723-740, doi: http://doi.org/10.12989/ sem.2013.45.6.723.

(24) Arenas, J.J., Aparicio, A.C. (1984). Estribos de puente de tramo recto. Santander: Universidad de Cantabria.

(25) Yepes, V., Díaz, J., González-Vidosa, F., Alcalá, J. (2009). Statistical characterization of prestressed concrete road bridge decks. Revista de la Construcción, 8(2): 95-109. http://www7.uc.cl/ccivil_revista/revista/rev15/REVISTA_15.pdf.

(26) Ministerio de Fomento. (2008). EHE-08 Instrucción de Hormigón Estructural. Boletín Oficial del Estado, n⿳00203. España.

(27) Soke, A., Bingul, Z. (2006). Hybrid genetic algorithm and simulated annealing for two dimensional non-guillotine rectangular packing problems. Engineering Applications of Artificial Intelligence, 19(5): 557-567, doi: http://doi. org/10.1016/j.engappai.2005.12.003. 
(28) Wu, T.H., Chung, S.H., Chang, C.C. (2009). Hybrid simulated annealing algorithm with mutation operator to the cell formation problem with alternative process routings. Expert Systems with Applications, 36(2): 3652-3661, doi: http://doi.org/10.1016/j.eswa.2008.02.06o.

(29) Martí, J.V., González-Vidosa, F., Yepes, V., Alcalá, J. (2013). Design of prestressed concrete precast road bridges with hybrid simulated annealing. Engineering Structures, 48: 342-352, doi: http://dx.doi.org/10.1016/j.engstruct.2012.09.014.

(30) Medina, J.R. (2001). Estimation of incident and reflected waves using simulated annealing. Journal of Waterway, Port, Coastal and Ocean Engineering, 127(4): 213-221, doi: http://dx.doi.org/10.1061/(ASCE)0733-950X(2001)127:4(213).

(31) Dowsland, K.A. (1993). Some experiments with simulated annealing techniques for packing problems. European Journal of Operational Research, 68(3), 389-399, doi: http://dx.doi.org/10.1016/0377-2217(93)90195-S.

(32) Dueck, G., Scheuer, T. (1990). Threshold accepting: A general purpose optimization algorithm superior to simulated annealing. Journal of Computation Physics, 90(1): 161-175, doi: http://dx.doi.org/10.1016/0021-9991(90)90201-B.

(33) Perea, C., Yepes, V., Alcalá, J., Hospitaler, A., González-Vidosa, F. (2010). A parametric study of optimum road frame bridges by threshold acceptance. Indian Journal of Engineering \& Materials Sciences, 17(6): 427-437, http://nopr. niscair.res.in/handle/123456789/10869. 